\title{
Caso exitoso de implementación de la metodología PADIE en la Facultad de Administración y Negocios
}

Eduardo Salazar Araújo

Ana Beatriz Blanco-Ariza

A lo largo de los capítulos que conforman este libro, se ha plasmado todo el recorrido que se ha tejido para llevar a cabo la implementación de la estrategia de articulación de la Docencia, Investigación, Extensión y Proyección Social. De esta manera, profesores y estudiantes en el aula de clase convergen ante diferentes temáticas y ejercicios que permiten durante su desarrollo realizar algunos productos derivados de esta estrategia; siendo posible alimentar los órganos de difusión de la facultad: revistas Desarrollo Gerencial y Liderazgo Estratégico.

Cada profesor al realizar la estrategia en el aula de clase selecciona los tres mejores productos elaborados por los estudiantes, estos son sometidos a evaluación y de allí se selecciona el mejor trabajo realizado. Una vez escogido este se postula a la revista estudiantil Liderazgo Estratégico, para que sea sometido a evaluación y posterior publicación. En ese orden de ideas, anualmente la revista publica los diferentes productos y resultados de estudiantes en compañía de los profesores.

Para dar mayor amplitud a los resultados obtenidos con la implementación de esta estrategia, a continuación, se presenta un ejercicio resultado de la aplicación de una línea de tiempo realizada 
por los estudiantes de primer semestre Jennifer Silva Tarra y Jhon López Jaramillo del Programa Administración de Empresas en la asignatura Fundamentos de Administración y Gerencia bajo la tutoría de la profesora Karelis Barrios Hernández.

\section{INFORME DE VISITA TÉCNICA}

El transporte marítimo es el principal medio de transferencia internacional de bienes y por supuesto es la vía de conexión más importante entre Colombia con el resto del mundo gracias a la privilegiada posición geográfica del país, por lo que la mayor parte del comercio exterior se realiza a través de este medio de transporte (Ballou, 2004).

Santa Marta es un puerto de suma importancia para el país, gracias a su ubicación geográfica, pues tiene unas condiciones naturales propicias para el desarrollo de la actividad portuaria, además de ser el puerto más profundo del continente americano y uno de los más seguros del mundo. Por lo tanto, muchas embarcaciones comerciales llegan a la ciudad apretando su desarrollo económico y el de la región Caribe colombiana (Legiscomex.com, 2014).

La Sociedad Portuaria de Santa Marta (s.f.) es la organización encargada de los puertos. Por medio de la información suministrada a partir de la visita al puerto se ha realizado el informe técnico, el cual enmarca las vivencias y conocimientos adquiridos durante el desarrollo de esta importante actividad académica.

El informe de visita se fundamenta en la guía de visita técnica empresarial, suministrada por el profesor, dentro de las actividades del programa PADIE para la asignatura de Comercio Internacional, en este trabajo se expone un análisis teórico de la industria portuaria, de igual manera un análisis del contexto industrial del puerto 
de Santa Marta, cumpliendo con el propósito que es contrastar la realidad del sector portuario con los conceptos desarrollados en clase.

Por otra parte, son expuestas la historia y evolución del puerto de Santa Marta, identificando las diferentes organizaciones e instituciones conexas al puerto, que realizan los distintos procesos que dan vida a la operación portuaria.

\section{ANÁLISIS TEÓRICO-CONCEPTUAL}

El trasnporte de mercancías es uno de los ejes centrales del comercio internacional. Anaya (2009) lo define como "toda actividad encaminada a trasladar los productos desde un punto de origen hasta un lugar de destino" (p.17). Es preciso aclarar que estos puntos deben encontrarse en lugares geográficos diferentes, puesto que toda movilización realizada dentro de un lugar de trabajo es considerada una manipulación de productos. El objetivo de la función de trasnporte es situar los productos en los puntos de destino correspondientes, bajo condiciones de seguridad en los tiempos adecuados y al menor costo.

De acuerdo con información publicada por el Trade \& Logistic Innovation Center (2011), "los puertos marítimos de un país constituyen uno de sus activos logísticos estratégicos más relevantes, dada su participación en el intercambio internacional de bienes". Un puerto marítimo está conformado por instalaciones provistas de espacios de aguas tranquilas que permiten la conectividad entre el medio marítimo y el terrestre, mediante la existencia de tres zonas principales: la zona marítima o de acceso, la zona terrestre para maniobras y la zona de enlace con los modos terrestres. 
De acuerdo con estadísticas de la Organización Mundial de Comercio ([OMC], 2015) más del $80 \%$ de las mercancías que se comercializan en el mundo se mueven por vía marítima, siendo los puertos los nodos que permiten operar dicho intercambio. En concordancia con esto, Castellanos (2009) considera que "de todos los modos de transporte, el transporte marítimo es el que mejor expresa la movilización internacional de carga, de acuerdo a su naturaleza" (p.73).

A pesar de su amplia utilización y las ventajas que ofrece el transporte marítimo al comercio internacional, esta modalidad de transporte presenta también algunas desventajas, las cuales se contrastan a continuación en la Tabla 7.

Tabla 7. Ventajas y desventajas del transporte marítimo

\section{VENTAJAS}

Capacidad: transporte en masa de grandes volúmenes, pues son los vehículos de transporte de mayor capacidad.

Competitividad: economías de escala debido a su gran capacidad y fuerza motriz; permite ofrecer tarifas de flete más bajas que cualquier otro modo.

Especialización: ofrece una gran variedad de buques para los distintos tipos de carga: carga general (suelta y unitarizada), carga a granel (líquidos: banqueros de diferentes tipos, sólidos: buques graneleros y buques de servicio combinado) y carga refrigerada.

Continuidad de las operaciones: en rango de 24 horas, es menos susceptible de sufrir variaciones, por condiciones climáticas adversas.

Flexibilidad: puede transportar diversos tipos de carga, pues no tiene restricciones para productos peligrosos.

\section{INCONVENIENTES}

Costos de embalaje: el manipuleo en puerto exige un embalaje de la carga resistente y relativamente costoso.

Velocidad: todos los demás modos de transporte son más rápidos, la duración promedio de la travesía es más larga que por aire o tierra. Esto repercute sobre los intereses y el monto del capital inmovilizado por inventario.

Accesibilidad: puertos marítimos están ubicados en sitios apartados de lugares de producción y destino final de las mercancías. Por tanto, es necesario transporte previo y posterior a la llegada de la carga al puerto, lo cual implica más manipuleo y mayor riesgo de daño. Cuando hay transbordo, el tiempo que la carga permanece en tránsito es más largo. Algunas rutas cierran en invierno por congelamiento.

Frecuencia de los servicios: el despacho de buques no ofrece tantas posibilidades como el servicio de carga aérea regular, transporte carretero o ferroviario. Menor frecuencia de servicios aumenta duración de almacenamiento de mercancía; baja rotación de inventarios.

Congestión portuaria: cada vez más frecuente, especialmente en países donde no existe una adecuación de las instalaciones portuarias paralela al aumento de su comercio exterior. 
Otro elemento importante que debe considerarse en la fundamentación teórica del presente trabajo es la carga, la cual de acuerdo con el Decreto 390 (2016) se puede definir como el "conjunto de mercancías que son objeto de una operación de transporte desde un puerto, aeropuerto, terminal terrestre o lugar de entrega, con destino a otro puerto, aeropuerto, terminal o lugar destino, amparadas en un documento de transporte" (p.6). En este sentido, también se debe considerar que la carga de acuerdo con su naturaleza se puede clasificar de la siguiente forma:

- Carga general: Todo tipo de carga de distinta naturaleza que se transporta conjuntamente, en pequeñas cantidades y en unidades independientes. Puede contarse con facilidad su número y en consecuencia se manipulan como unidades. Se transportan y se almacenan juntas.

- Carga a granel: Es la carga que se transporta en abundancia y sin necesidad de embalaje o unitarización; se estiba directamente en la bodega de los buques en grandes compartimientos especialmente acondicionados. La carga a granel puede ser: líquida, sólida o gaseosa y generalmente se miden en unidades de peso o volumen. Esta carga por lo general se almacena en tanques o silos.

- Carga especial: Es aquella carga que por su naturaleza posee características diferentes de las demás, generadas por varios factores como el peso, el grado de conservación, su peligrosidad, alto valor, o tamaño, entre otros, por lo cual su manipulación requiere de un cuidado especial para su estiba.

\section{ANÁLISIS DE CONTEXTO INDUSTRIAL}

La Sociedad Portuaria de Santa Marta (s.f.) "nace con la Ley 001 de 1991, norma que finalizó el monopolio estatal en la administración portuaria y que llevó a la liquidación de Colpuertos", lo que ha 
dado paso a la creación de nuevas entidades del orden público, como la Superintendencia General de Puertos, abriendo el camino a la inversión privada a las sociedades portuarias regionales y los operadores portuarios inició sus operaciones en el año de 1993.

La Sociedad Portuaria de Santa Marta (s.f.) en sus comienzos estaba conformada por empresas de las regiones del Urabá y Magdalena, navieras y empresas del sector público del departamento del Magdalena, convirtiéndose en una sociedad mixta. Actualmente, el puerto ha ido creciendo de manera muy rápida, caracterizándose por la eficiencia en su manejo de cargas y su portafolio de productos y servicios ha incrementado significativamente, maneja productos como: granel sólido, líquido y carbón, este último representa la mayor parte de ingreso en el puerto; constituye un puerto de gran importancia para la economía colombiana, y a nivel de comercio exterior maneja 6.000 toneladas de mercancía.

La Sociedad Portuaria de Santa Marta (s.f.) es una concesión que cuenta con autonomía administrativa y patrimonio propio para la administración y operación de la infraestructura del Terminal Marítimo.

El puerto cuenta con siete muelles en los que presta sus servicios las 24 horas, ofrece una terminal de carga general, terminal de carbón, terminal de granel y terminal de contenedores, dispone de una excelente infraestructura y servicios logísticos, para almacenaje seguro y práctico en cada una de sus bodegas y patios. Además, es el único de la costa Atlántica que cuenta con modalidad de transporte ferrocarril, que posibilita generar cargues y descargues directos, lo que facilita el manejo de carga a granel. A continuación en la Figura 3 se exponen los elementos: misión y visión del direccionamiento estratégico de la Sociedad Portuaria. 


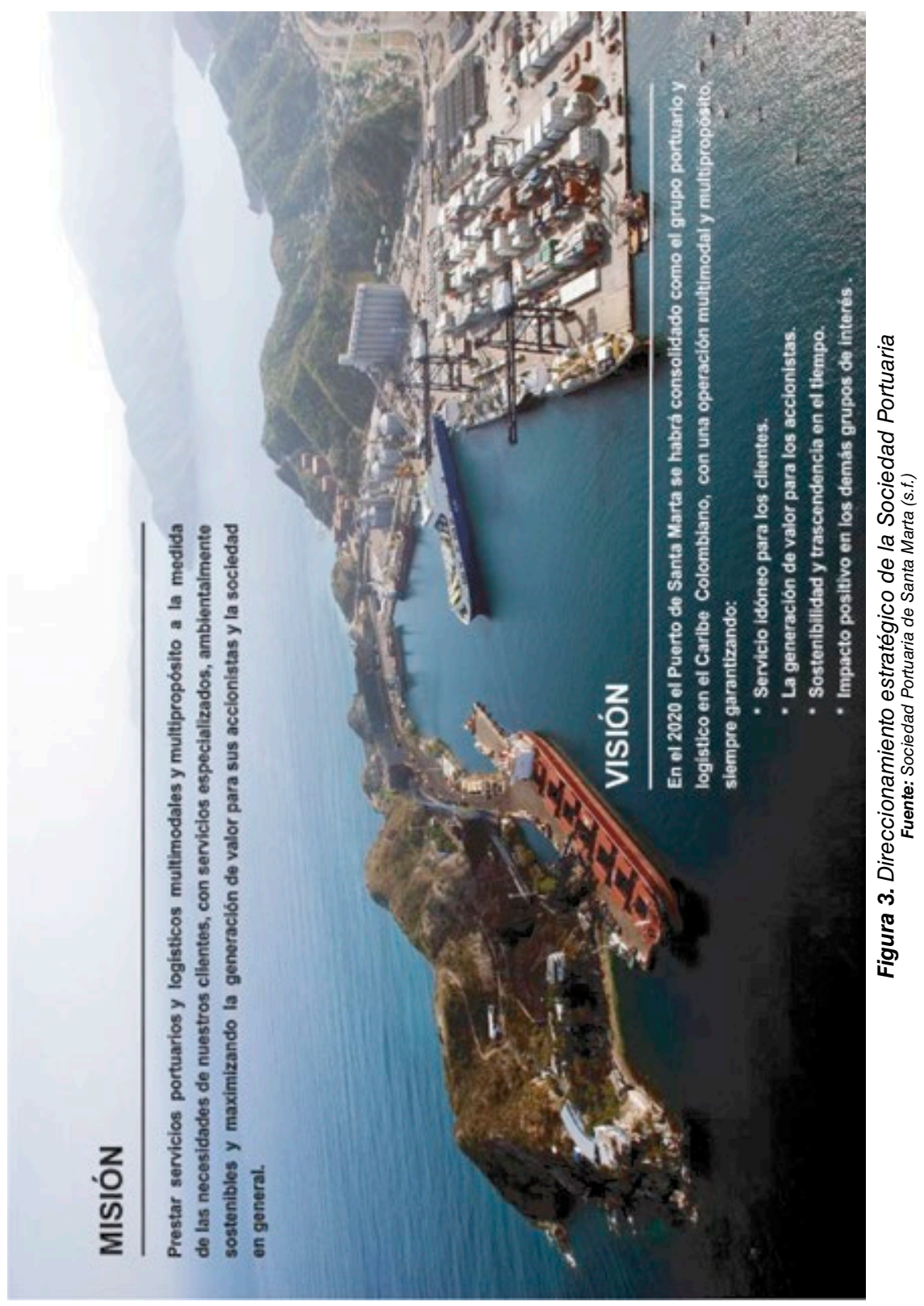


El puerto de Santa Marta se ubica en la bahía de la misma ciudad departamento del Magdalena, al norte de Colombia. Con el fin de georreferenciar su localización a continuación en la Figura 4 se puede apreciar la ubicación exacta del puerto.

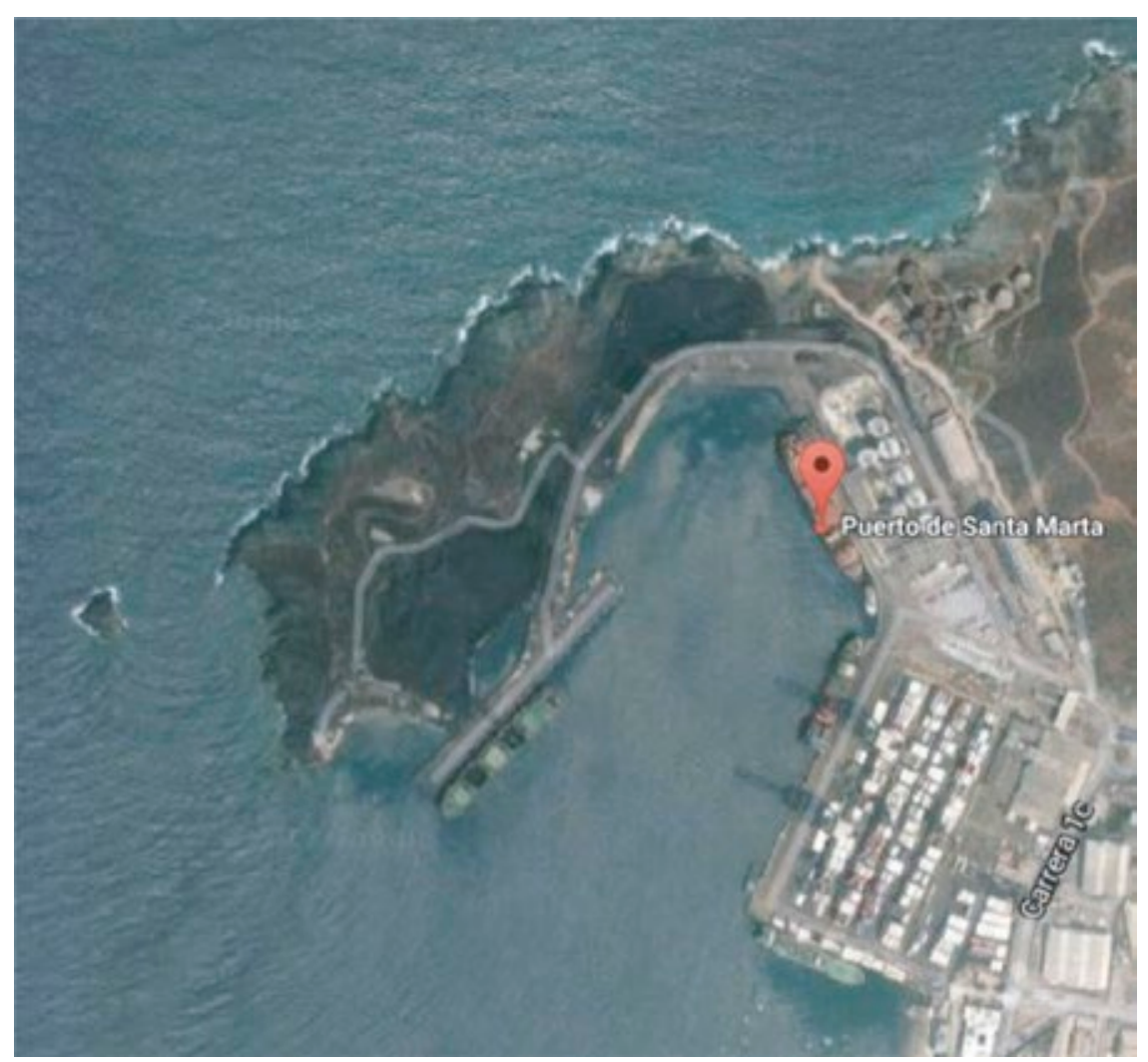

Figura 4. Vista satelital del puerto de Santa Marta

Fuente: Consulta realizada por Google Maps (2016)

A partir de su localización, se afirma que su ubicación natural a orillas de una profunda bahía en el mar Caribe ofrece condiciones óptimas para facilitar el acceso y operación en las diferentes áreas colindantes al territorio, lo que permite el ingreso de buques de gran calado, y exige planificación de la capacidad instalada del puerto. 
De acuerdo con la información suministrada durante la visita por Alejandra García, funcionaria del departamento comercial de la Sociedad Portuaria de Santa Marta, se destacó que esta terminal portuaria fue superior en tiempo de movilización a los demás puertos en el cargue de contenedores, al movilizar 72,4 contenedores por hora y 490 movimientos en un período de solo nueve horas, lo que estableció un récord en las operaciones portuarias.

Igualmente, en carga a granel se logró el descargue de 26.000 toneladas de maíz y trigo en solo 37 horas (operación que normalmente dura entre 55 y 60 horas) por medio de una completa infraestructura compuesta por silos y bodegas mecanizadas para el almacenamiento, lo cual es muy valorado por sus usuarios, pues en logística el tiempo es uno de los recursos más valiosos. Los principales servicios ofrecidos por la Sociedad Portuaria de Santa Marta están orientados a los operadores portuarios marítimos y terrestres, a operadores de comercio exterior y servicios a la carga, dentro de estos servicios se destacan:

- Muellaje para líneas regulares y no regulares

- Cargue y descargue de mercancía a granel

- Cargue y descargue de contenedores

- Trasbordo nacional e internacional

- Exportaciones, importaciones y cabotaje

- Suministro de energía eléctrica y agua potable

- Almacenaje de contenedores, vehículos y mercancía a granel.

Unos de los objetivos centrales del puerto de Santa Marta es incrementar las operaciones de exportación, actividad en la cual 
pretende ganar una mayor participación en el mercado. El puerto compite con todos los puertos ubicados en el mar Caribe, aunque su infraestructura permite la manipulación de cualquier tipo de carga, la Sociedad Portuaria de Santa Marta es reconocida por ser uno de los puertos más seguros para el almacenamiento, transporte, cargue y descargue de mercancía a granel, para lo cual cuenta con grúas especializadas y silos para el almacenamiento de este tipo de carga.

Los principales productos que se exportan desde el puerto son banano, café y granos, hasta maquinaria pesada y carbón. Su entorno y su compromiso ambiental han sido factores determinantes para que la Sociedad Portuaria de Santa Marta implemente políticas para la reducción de los impactos ambientales de su operación.

El puerto de la ciudad de Santa Marta está conectado con todos los mercados, de manera directa o con los puertos de trasbordo internacional. Colombia hace negocios con Estados Unidos, Centroamérica y el Caribe, y particularmente de fruta y carbón con Europa norte; las exportaciones hacia Sudamérica son muy pocas.

En el 2013 se redujo en más del 30 \% el tiempo en atención de motonaves, se generaron más de 300 empleos entre directos e indirectos y hubo un incremento en las exportaciones e importaciones. En cuanto a las ventas externas, el puerto obtuvo un crecimiento del $26 \%$ en la movilización de carga, especialmente de frutas refrigeradas, entre las que se encontraban el banano y la uchuva. Por su parte, el aguacate y el aceite vegetal también tuvieron un importante incremento del $17 \%$ y el $54 \%$, respectivamente. En el caso de las compras internacionales, hubo un crecimiento del $13 \%$ en 
carga suelta, como el acero y la tubería; un $40 \%$, en infraestructura vial, y un $16 \%$, en granel. Las bajas condiciones de humedad y salinidad del puerto fueron características claves para aumentar la compra de vehículos a través de esta terminal, la cual aumentó en un $5 \%$ respecto al 2012.

Mediante cuatro terminales portuarias, constituidas por el puerto de Santa Marta, Prodeco (filial de Glencore), Drummond y Colombia Natural Resources se mueven millones de toneladas de carbón al año. En el puerto se manejan normas de protección industrial que cuidan a todos los trabajadores y a las personas que lo visitan también se aplican normas de seguridad integral, y normas de seguridad ambiental por las cuales ha recibido reconocimientos.

La eficiencia en el aprovechamiento del agua, la utilización de equipos eléctricos en reemplazo de equipos con gasolina y diesel, trabajo social y una operación de carbón sostenible, resultaron factores relevantes en el proceso de renovación de la certificación llevado a cabo por LloydsRegister y ECO SLC. El puerto de Santa Marta, aparte de ser el primer puerto por fuera de Europa en haber sido certificado hace tres años con la prestigiosa acreditación ambiental europea ECOPORTS, hoy se convierte en el primer puerto por fuera del viejo continente, en lograr por segunda ocasión certificarse en esta misma norma.

La responsabilidad social empresarial de la Sociedad Portuaria de Santa Marta (s.f.) está orientada a satisfacer las necesidades y expectativas de estos grupos, a través de proyectos sostenibles direccionados al mejoramiento de la calidad de vida, educación, preservación del medio ambiente y su entorno, entre sus valores de responsabilidad social están: Ecoeficiencia: ser ecologista, El mejor lugar para trabajar, Empresa ciudadana: ser buen vecino. 
Hay varias empresas que operan actualmente en el puerto entre las cuales está: su empresa filial OPERLOG, ofrece servicios especializados para la atención de la carga brindando servicios de descargue y cargue de motonaves, porteo y cargue para el despacho de mercancías; Carbosan, es la filial de la Sociedad Portuaria de Santa Marta (s.f.), encargada de realizar las operaciones de carbón al interior del terminal samario; International Terminal Company S.A. es una sociedad entre SSA International y la Sociedad Portuaria creada con el fin de administrar, operar y comercializar el terminal de contenedores del puerto de Santa Marta, todas estas con certificaciones de calidad.

La Sociedad Portuaria de Santa Marta (s.f.) ha logrado varios reconocimientos por el buen trabajo que viene realizando, además de la certificación ECOPORTS, se logró un récord en la operación de la motonave Bosporus Highway de la línea K-Line procedente de México, con 632 carros marca Chevrolet pertenecientes a General Motors, logrando una productividad de descargue por hora de 332 unidades por medio de operación rodada, superando la media continental que es de 160 vehículos por hora y el récord propio establecido en América, que era de 276 unidades por hora.

Anteriormente tuvimos una eficiencia de 272 vehículos por hora que de por sí ya era un récord continental, ahora estamos batiendo nuestro propio récord logrando 332 unidades descargadas por hora, por ende, esto significa más oportunidades de empleo para nuestra gente. (El Tiempo, 2015)

Según el reporte del puerto samario, esta operación se desarrolló con cero incidentes,

Por lo que adicionalmente en términos de ahorro significa menos costo que asume la naviera en muellaje y asistencia en puerto, 
por lo que resulta atractivo volver a recalar en el Puerto de Santa Marta, así como el ahorro que representa para el propietario de la carga maximizando su entrega en tiempo al destinatario final. (El Tiempo, 2015)

Con el fin de informar y contextualizar respecto a la infraestructura del puerto de Santa Marta, a continuación se exponen fotografías de los tres principales lugares detallados en la visita.

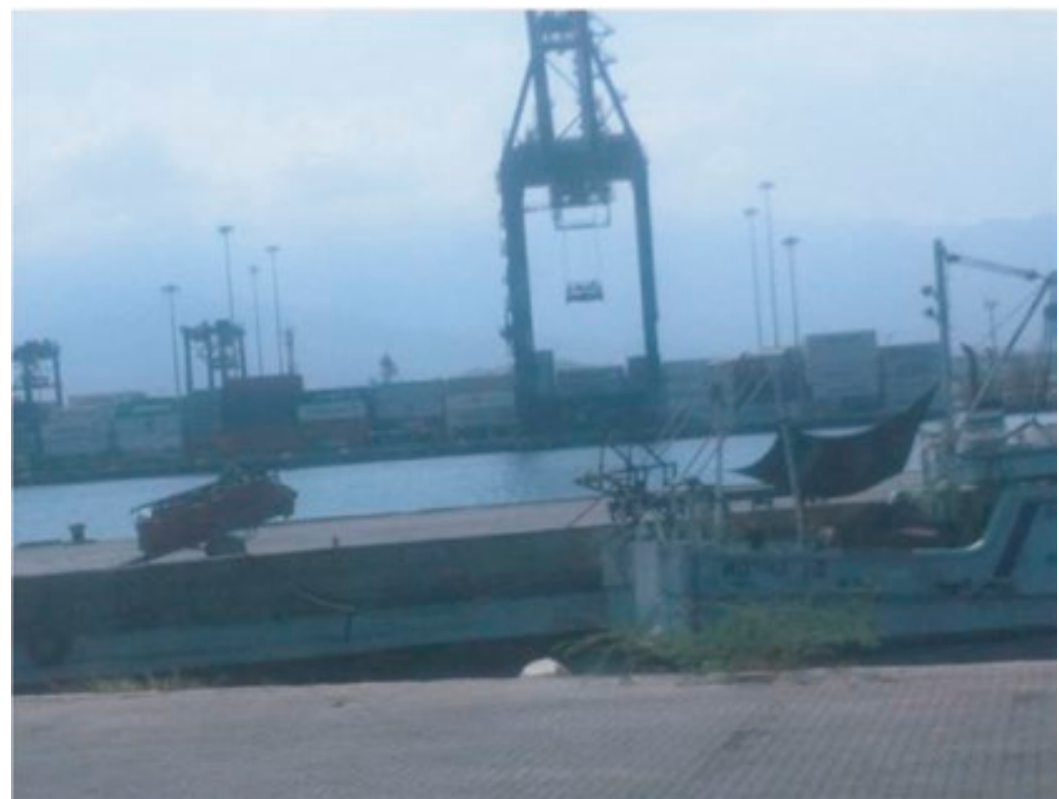

Fotografía 1. Patio de contenedores

Fuente: Elaboración propia 
Programa de Articulación de las Funciones de Docencia, Investigación, Extensión y Proyección Social (PADIE). Un enfoque desde la Facultad de Administración y Negocios

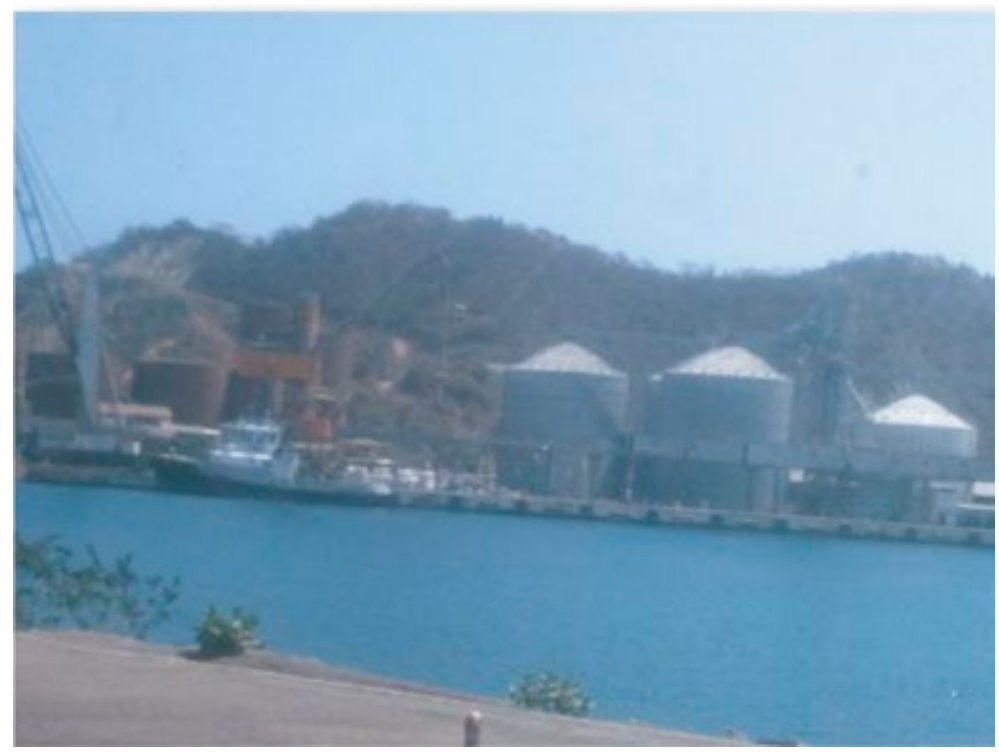

Fotografía 2. Silos de almacenamiento de carga

Fuente: Elaboración propia

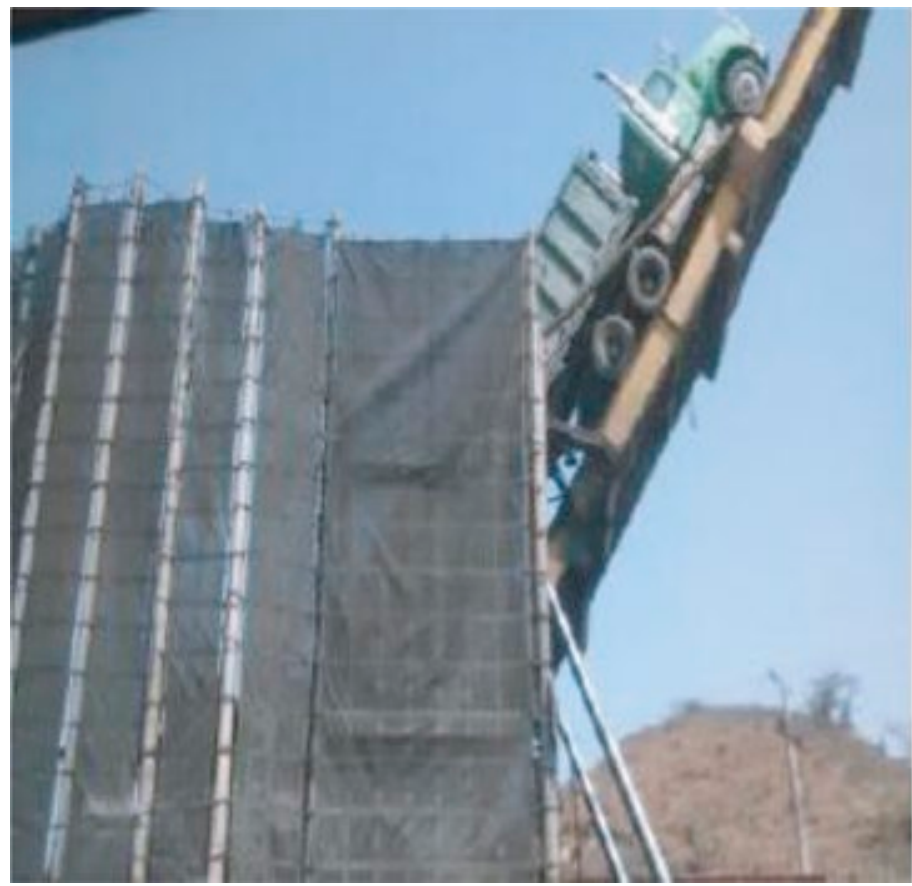

Fotografía 3. Proceso de cargue de carbón

Fuente: Elaboración propia 


\section{REFLEXIONES FINALES DE LA VISITA EMPRESARIAL}

Después de realizar un estudio del estado actual del puerto de Santa Marta, se hace evidente como han invertido y han cambiado la estructura y operativos con el fin de alcanzar un mejor posicionamiento competitivo a nivel internacional. Uno de los mayores éxitos que ha logrado es la infraestructura vial, lo cual está directamente relacionado con la inversión y es un correcto aprovechamiento de los recursos del sector.

El buen funcionamiento de las vías garantiza el eficiente flujo de entrada de los camiones hacia las zonas portuarias y gracias a esto se reduce el tiempo de entrega de las mercancías de manera impactante; no solo se ha invertido en las vías, sino en las maquinarias que producen el doble por horas, lo que es un avance provechoso para todos los colombianos que permitirá impulsar la imagen y el posicionamiento en ranking a nivel mundial.

Uno de los desafíos más grandes que enfrenta el país es adecuar la tecnología y la capacidad de los puertos. El puerto de Santa Marta está mejor posicionado gracias a su maquinaria moderna y la profundidad del agua que permite que los buques puedan llegar y desembarcar con más facilidad, esta ampliación representa para Colombia una reconfiguración del tráfico marítimo y una oportunidad para aumentar el flujo de comercio exterior.

Para obtener beneficio de estos cambios, la nación se ve en la necesidad de adecuar sus terminales portuarias y aumentar la capacidad instalada con el fin de brindar atención a la carga mientras se encuentra en el puerto. Todos estos cambios generarían una mayor eficiencia en el sector portuario en Colombia, lo cual se traduce en una mayor agilidad en los procesos, menos costos 
de transacción-transporte y más agilidad en los procesos operativos, con esto se aclara que se ha avanzado más en el puerto de Santa Marta y aún se requiere definir e implementar cambios en puertos como Buenaventura, especialmente en sus vías y en la estructura, también en los puertos de las ciudades de Cartagena y Barranquilla.

Otro punto fuerte es la implementación de procesos que permiten que el puerto se convierta en el único en Colombia con certificación Ecoports. Para lograr ese reconocimiento fue necesario apostarle a la responsabilidad social e invertir recursos.

De esa forma se puede que concluir que el puerto de Santa Marta se está proyectando en ser uno de los mejores puertos de Colombia, gracias a la buena gestión de sus dirigentes, que están aprovechando los recursos y capacidades que tiene la ciudad y aportan cada año más en la economía nacional.

Finalmente, se afirma que el transporte marítimo aventaja al terrestre y al aéreo, por su mayor capacidad, por su bajo coste y por la flexibilidad de su utilización; abarcando una actividad internacional que permite a cualquier empresa acceder a mercados externos independientemente de su nacionalidad y de su ubicación; y que la eficiencia de la actividad portuaria impulsa el desarrollo económico de las naciones y genera mayor competitividad para el sector productivo de un país.

\section{IMPACTO DE LA ESTRATEGIA PADIE}

Así, los estudiantes durante cada semestre realizan un ejercicio que implica relacionar la investigación a lo visto en las asignaturas, para este caso particular por ser estudiantes de primer semes- 
tre, realizaron como ejercicio una línea de tiempo que permitiera conocer la evolución de la administración en la historia y a la vez, el desarrollo de este ejercicio lo plasmaran en un ensayo para poder ser utilizado como medio de consulta para otros estudiantes de su mismo programa.

En ese sentido, el estudiante empieza a hacer uso de las bases de datos con que cuenta la universidad, aprende a utilizar las normas APA y a realizar fichas bibliográficas y de consulta que terminarán siendo su herramienta a partir de ese momento para la consecución de información relevante en cada actividad que realice. Así también conoce más de la disciplina que estudia y desde sus inicios se foguea con escenarios que le permiten demostrar los conocimientos adquiridos, además de generarle confianza al hablar en público y dominio propio a la hora de enfrentarse a cualquier escenario donde tenga que exponer sus ideas, esto como parte también de la formación integral que se profesa al estudiantado.

No obstante, el profesor juega un rol muy importante dentro de este proceso dado que es él quien ayuda y refuerza las potencialidades que tiene cada uno de los estudiantes dentro del aula de clase. De esta manera, estudiantes y profesores convergen en el estudio de un mismo tema y en el desarrollo de documentos que sirven como material de estudio y consulta de los estudiantes.

\footnotetext{
Cómo citar este capítulo:

Salazar Araújo, E. \& Blanco-Ariza, A. (2017). Caso exitoso de implementación de la metodología PADIE en la Facultad de Administración y Negocios. En: Blanco-Ariza, A. \& Rodríguez-Calderón, G. (coord.). Programa de Articulación de las Funciones de Docencia, Investigación, Extensión y Proyección Social (PADIE). Un enfoque desde la Facultad de Administración y Negocios. (pp.69-85). Barranquilla, Colombia: Ediciones Universidad Simón Bolívar.
} 